\title{
Analysis of a Plasmonic Based Optical Fiber Optrode With Phase Interrogation
}

\author{
H. MOAYYED ${ }^{1 *}$, I. T. LEITE ${ }^{1}$, L. COELHO ${ }^{1}$, J. L. SANTOS $^{1}$, and D. VIEGAS ${ }^{2}$ \\ ${ }^{1}$ CAP / INESC TEC - Technology and Science and Faculty of Sciences from the University of Porto, Rua do Campo \\ Alegre 687, 4169-007 Porto, Portugal \\ ${ }^{2}$ INL - International Iberian Nanotechnology Laboratory, Av. Mestre José Veiga, 4715-310 Braga, Portugal \\ ${ }^{*}$ Corresponding author: H. MOAYYED $\quad$ E-mail: hmoayyed@fc.up.pt
}

\begin{abstract}
Optical fiber optrodes are attractive sensing devices due to their ability to perform point measurement in remote locations. Mostly, they are oriented to biochemical sensing, quite often supported by fluorescent and spectroscopic techniques, but with the refractometric approach considered as well when the objective is of high measurement performance, particularly when the focus is on enhancing the measurand resolution. In this work, we address this subject, proposing and analyzing the characteristics of a fiber optic optrode relying on plasmonic interaction. A linearly tapered optical fiber tip is covered by a double overlay: the inner one - a silver thin film and over it a dielectric layer, with this combination allowing to achieve, at a specific wavelength range, surface plasmonic resonance (SPR) interaction sensitive to the refractive index of the surrounding medium. Typically, the interrogation of the SPR sensing structures is performed, considering spectroscopic techniques, but in principle, a far better performance can be obtained, considering the reading of the phase of the light at a specific wavelength located within the spectral plasmonic resonance. This is the approach which is studied here in the context of the proposed optical fiber optrode configuration. The analysis performed shows the combination of a silver inner layer with a dielectric titanium oxide layer with tuned thicknesses enables sensitive phase reading and allows the operation of the fiber optic optrode sensor in the third telecommunication wavelength window.
\end{abstract}

Keywords: Optrode; fiber sensing; surface plasmon resonance; phase interrogation; metallic-oxide coupled layers

Citation: H. MOAYYED, I. T. LEITE, L. COELHO, J. L. SANTOS, and D. VIEGAS, "Analysis of a Plasmonic Based Optical Fiber Optrode With Phase Interrogation," Photonic Sensors, 2016, 6(3): 221-233.

\section{Introduction}

Along with the years, a large number of fiber based sensing head layouts have been proposed to measure a broad range of parameters, relying on diverse interaction mechanisms of the measurand with the optical field [1]. One of the most attractive layouts is the reflective fiber tip configuration, where the sensor functionality occurs at the end of the optical fiber, therefore readily allowing point sensing at remote locations. These fiber sensing devices are commonly identified as fiber optrodes, a generalization of the initial meaning of the word optrode, and the combination of the words "optics" and "electrode" indicates it strictly refers to the modulation of the optical field by the electric characteristics of an electrode which interacts with the targeted measurand [2]. To a large degree, fiber optrodes include a membrane at the tip of the fiber

Received: 18 March 2016 / Revised: 6 June 2016

(C) The Author(s) 2016. This article is published with open access at Springerlink.com

DOI: $10.1007 / \mathrm{s} 13320-016-0329-5$

Article type: Regular 
with its fluorescent and spectroscopic properties changing with the variation of the measurand (typically a chemical or biochemical entity), inducing an intensity/spectral modulation of the light that returns along the fiber which is also the illumination channel [3]. However, other operation modes are possible, and the one associated with refractometry has the particular importance, where the sensing head is sensitive to the refractive index of the surrounding medium, which by itself can show a specific dependence on a certain chemical or biochemical parameter [4].

Since its first observation by Wood in 1902 [5], the physical phenomenon of plasmonics has been found its way into practical applications in sensitive detectors, capable of detecting sub-monomolecular coverage. Physical interpretation of the phenomenon was initiated by Lord Rayleigh [6] and further refined by Fano [7], but a complete explanation was not possible until 1968, when Otto [8] and in the same year Kretschmann and Raether [9] reported the excitation of surface plasmons. Nowadays, plasmonics is one of the most active research topics in photonics. The fundamental reason behind the ever-growing importance of plasmonics in today's scientific research lies on the subjacent physical phenomenon, which is an interaction process between the electromagnetic radiation and the conduction electrons at metallic interfaces or in small metallic nanostructures, leading to an enhanced optical near-field of the sub-wavelength dimension. Physically, this arises from the fact that the length scale involved in the mode coupling between photons and electrons has an effective value much smaller than the light's wavelength, essentially determined by the electron dynamics in the metal, with the light energy carried as a package of electron oscillations [10].

Owning to the well-known advantages over conventional sensors, surface plasmon resonance fiber optic sensors have attracted much attention in the past two decades. The importance of surface plasmonic resonance (SPR) fiber optic sensors in the field of chemical, biological, and environmental sensing has notably increased in the last years [11-12]. Indeed, ever since the introduction to various optical methods in the excitation of the SPR at a metal-dielectric interface [13], it has been widely recognized that such effect can be utilized to achieve sensing or monitoring of various interfacial phenomena with ultrahigh sensitivity. These include, for example, chemical and biological sensing [14, 15], film-thickness sensing [16], temperature sensing [17], and angular measurement [18]. Recently, it has been demonstrated that the SPR technique applied to chemical and biological sensing can achieve refractive index (RI) resolutions down to $10^{-9}$, values not accessible to other optical sensing technologies [19].

The light polarization can be TE or TM polarization, although only the TM polarization will excite the plasmon in a waveguide, which is the condition achieved looking for the phase matching between a plasmon and a waveguide mode, mathematically meaning the equality of the modal propagation constants of the plasmon $\left(k_{\mathrm{SP}}\right)$ and the optical waveguide mode ( $\left.k_{\text {waveguide }}\right)$, which corresponds to the equality of their effective refractive indices. In general, there are four approaches to interrogate an SPR sensing structure, namely (1) the change in the resonant angle ("angular interrogation"), (2) the change in reflectance at the fixed incidence angle ("intensity interrogation"), (3) the change in the resonant wavelength at the fixed incidence angle ("wavelength interrogation"), and (4) the phase difference between the p- and s-polarization states of the reflected light ("phase interrogation") [15]. The plasmonic based modulation in an optical fiber does not rely on the incidence angle of the light, as in the Otto or Kretschmann-Raether configurations, but instead on the modulation of the intensity, wavelength or phase of the light, i.e., the output signal can be obtained by taking into account the 
total power transmitted through the device, analyzing the spectral properties of the transmitted light or by reading and processing the phase of this light.

In phase interrogation of SPR sensors, there is a lack of comprehensive analysis. Surely, this methodology is somewhat more complex to implement than most traditional ones, requiring deeper know-how on optical components, modulation approaches, and signal processing techniques, but eventually, the main reason for this situation is the circumstance: the great majority of the R\&D (research and development) communities which has been working until recently in SPR sensing have a biochemical background, therefore with a tendency to use the spectroscopic equipment. This is changing since plasmonics is moving to fields other than sensing, such as imaging and data storage, attracting the subject researchers with diverse backgrounds. A consequence of this dynamics is a clear improvement of the resolution values obtained when considering phase interrogation. Indeed, for the case of heterodyne interrogation (when the TE and TM waves are provided by two light sources with slightly different optical frequencies that are made to interfere, resulting in a beat signal with the frequency low enough to be resolved by the photodetection and amplification electronics), a value of $2.8 \times 10^{-9}$ was reported in 2008 [19], which seems to be the highest refractive index resolution achieved so far; when considering polarimetric interrogation (a polarizer is used to obtain, from a single optical source, TE and $\mathrm{TM}$ components which interfere on the photodetector), and under the optimized conditions, a resolution of $3.7 \times 10^{-8}$ was obtained [20]; applying the methodology of the reference wave propagating along a different spatial path, resulting in interfering with the TM light on the photodetector, a resolution down to $2.2 \times 10^{-7}$ was reported in 2011 [21].

Considering optical fiber sensing platforms, phase interrogation is attractive since the SPR induced phase change is greatly amplified due to the multiple attenuated total reflections undergone by light in the fiber, with a consequent increase in measurand readout sensitivity. This factor certainly contributes to the interest this interrogation approach has been inducing in recent years [22].

There are several methodologies to identify the performance of an SPR optical fiber sensing system. One of the most common methods is by evaluating its intrinsic sensitivity [23, 24]. Focusing on this characteristic, several attempts have been considered, such as tapering optical fibers [25]. For instance, a tapered optical fiber SPR microsensor based on white light excitation [26] and the use of dual-tapered and tetra-tapered fiber SPR probes for gas and liquid sensing have been proposed [27]. These structures present a substantial variation in evanescent field penetration along the tapered sensing region length. The evanescent field can be reached by the narrowing of the fiber cross section in the tapered region. Another approach, compatible with the mentioned mechanisms, is based on modifying the geometry of the sensing head (tip layout) to optimize the dynamic range and sensitivity [28-30]. These configurations are supported by beveling the distal end of the fiber probe, such as a chisel-tipped probe, where the top and bottom of the probe are polished to the same angle.

In this work, the combination "optrode + refractometry associated with plasmonics + phase interrogation" is analytically explored. A linearly tapered fiber tip coated with two thin film layers is considered: the inner one with a metallic material as silver (Ag) and the external layer with a dielectric oxide. This combination permits to achieve plasmonic resonance at a specific wavelength window sensitive to refractive index variations of the external medium. Insights into the experimental implementation and characterization of this SPR sensing structure are also delivered. 


\section{Sensor layout and analysis}

Figure 1 shows an optical fiber tip optrode with the linear profile, in which the conical surface is coated with a reflective thin film. The tip length is assumed to be $L, \rho_{0}$ and $\rho_{\mathrm{L}}$ denote the radii of the fiber core and tip end, respectively. A geometric ray methodology combined with the transfer-matrix formalism for stratified optical media is applied here, following the approach of Gupta and co-workers $[23,25]$.

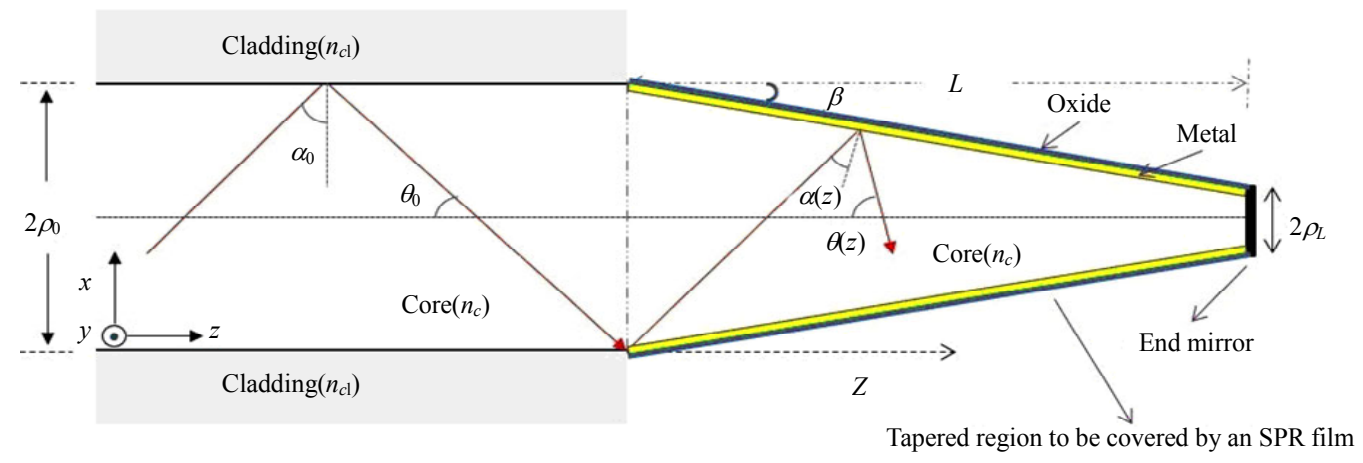

Fig. 1 Schematic illustration of the SPR fiber optrode under analysis (the top and bottom of the probe are polished to the same angle; the cladding of the optical fiber tip has been removed in the sensing region and replaced by a metallic film covered with an oxide overlayer).

If $\theta_{0}$ represents the propagation angle that the optical ray makes with the axis of the fiber, it is transformed to an angle $\theta(z)$ inside the taper following the transformation relationship:

$$
\rho_{0} \sin \theta_{0}=\rho(z) \sin \theta(z)
$$

where $\rho(z)$ is the taper radius at a distance $z$ from the input end of the taper $(z=0)$. In the taper region, such optical ray reaches the interface with the metal-dielectric film at an angle of incidence, and $\alpha(z)$, at coordinate $z$, is related with the value $\alpha_{0}$ in the non-tapered region as

$$
\alpha(z)=\cos ^{-1}\left[\frac{\rho_{0} \cos \alpha_{0}}{\rho(z)}\right]-\beta
$$

where $\rho(z)=\rho_{0}-(z / L)\left(\rho_{0}-\rho_{L}\right)$ is the tip radius with the coordinate $z$, and $\beta=\tan ^{-1}\left(\left(\rho_{0}-\rho_{L}\right) / L\right)$ is the taper angle. Notice that as the radius $\rho(z)$ decreases, so does the angle $\alpha(z)$. Therefore, for a particular optical ray launching at the input end of the taper to undergo total internal reflection throughout the entire length of the taper, the condition $\alpha(z)>\alpha_{c}=\sin ^{-1}\left(n_{s} / n_{\mathrm{co}}\right)$ must be satisfied for $0<z<L\left(n_{s}\right.$ and $n_{\mathrm{co}}$ are the refractive indices of the surrounding medium and fiber core, respectively). Since $\alpha(z)$ becomes smaller along the propagation and considering (2), the above condition is fulfilled when

$$
\cos ^{-1}\left(\frac{\rho_{0} \cos \alpha_{0}}{\rho_{L}}\right)-\beta>\alpha_{c} .
$$

This is the basic equation which permits to analyze the two relevant situations, namely for a fixed angle $\alpha_{0}$ what is the maximum length of the optrode (taper) to keep the condition of total reflection along its length and the other way around, i.e., for a fixed taper length what is the minimum value for $\alpha_{0}$.

Considering the first of these two situations, regarding (3), and assuming a fixed angle of incidence, the maximum taper length is

$$
L>\frac{\rho_{0}-\rho_{L}}{\tan \left[\cos ^{-1}\left(\frac{\rho_{0} \cos \alpha_{0}}{\rho_{L}}\right)-\alpha_{c}\right]} .
$$

On the other hand, when $L$ is fixed, the minimum angle of incidence must satisfy

$$
\alpha_{0}>\cos ^{-1}\left[\frac{\rho_{L}}{\rho_{0}} \cos \left[\beta+\alpha_{c}\right]\right] .
$$

The angle range of the rays at the coordinate $z$ alters to $\left[\alpha_{1}(z), \alpha_{2}\right]$ due to the variation of the fiber core diameter, where

$$
\alpha_{1}(z)=\cos ^{-1}\left[\frac{\rho_{0} \cos \alpha_{\mathrm{cr}}}{\rho(z)}\right]-\beta
$$




$$
\alpha_{2}=\frac{\pi}{2}-\beta
$$

where $\theta_{\mathrm{cr}}=\sin ^{-1}\left(n_{\mathrm{cl}} / n_{\mathrm{co}}\right)$ is the critical angle $\left(n_{\mathrm{cl}}\right.$ and $n_{\mathrm{co}}$ are the refractive indices of the fiber cladding and core, respectively) [30].

In order to evaluate the optical response of the sensing structure, we use the multilayer transfer-matrix theory applied to a four-layer system comprising: (1) fiber-core, (2) inner-metal, (3) oxide-layer, and (4) probe-medium [23]. This methodology is applied to compute the propagation of the radiation through the layer system using Maxwell's equations subject to boundary conditions between two adjacent layers. From the basis of this formalism, the tangential components of the electric and magnetic field vectors ( $\mathbf{E}$ and $\mathbf{H}$, respectively) at the first and final boundaries, are related with [31]

$$
\left[\begin{array}{l}
U_{0} \\
V_{0}
\end{array}\right]=\left[\begin{array}{ll}
m_{11} & m_{12} \\
m_{21} & m_{22}
\end{array}\right]\left[\begin{array}{l}
U_{N} \\
V_{N}
\end{array}\right]
$$

where $(U, V)=\left(E_{y}, H_{z}\right)$ for the TE modes or $(U, V)=\left(H_{y},-E_{z}\right)$ for the TM modes, and $\left[m_{i j}\right]$ is the transfer matrix of the multilayer structure comprised of $N$ interfaces:

$$
\left[\begin{array}{ll}
m_{11} & m_{12} \\
m_{21} & m_{22}
\end{array}\right]=\prod_{k=1}^{N-1}\left[\begin{array}{cc}
\cos \delta_{k} & \frac{-\mathrm{i}}{\eta_{k}} \sin \delta_{k} \\
-\mathrm{i} \eta_{k} \sin \delta_{k} & \cos \delta_{k}
\end{array}\right]
$$

with $\delta_{k}$ being the phase in the $k$ th layer $(k=0$ and $k=N$ are the first and final media, respectively), given by

$$
\delta_{k}=\frac{2 \pi}{\lambda} n_{k} \cos \theta_{k} d_{k}=\frac{2 \pi d_{k}}{\lambda} \sqrt{\varepsilon_{k}-n_{0}^{2} \sin ^{2} \theta_{0}}
$$

where $d_{k}, n_{k}$, and $\theta_{k}$ are, respectively, the thickness, refractive index, and propagation angle (with respect to the normal to the direction of stratification) of the $k$ th layer, $\lambda$ is the free-space wavelength of the light propagating in the system, and non-magnetic media are assumed (i.e., $\mu_{k}=1$ ). The parameter $\eta_{k}$ in (9a) is defined as a function of the polarization state as

$$
\eta_{k}^{s}=\sqrt{\frac{\varepsilon_{0} \varepsilon_{k}}{\mu_{0} \mu_{k}}} \cos \theta_{k} \quad \text { (for TE waves) }
$$

and

$$
\eta_{k}^{p}=\sqrt{\frac{\mu_{0} \mu_{k}}{\varepsilon_{0} \varepsilon_{k}}} \cos \theta_{k}=\frac{\mu_{0} \mu_{k}}{\varepsilon_{0} \varepsilon_{k}} \eta_{k}^{s}(\text { for TM waves)(10b) }
$$

where $\varepsilon_{k}$ and $\mu_{k}$ are the dielectric constant and relative magnetic permeability, respectively. The reflectance $R_{s, p}$ of the multilayer structure for each polarization state is obtained as

$$
R_{s, p}(\lambda)=\left|r_{s, p}(\lambda)\right|^{2}
$$

where $r_{s}$ and $r_{p}$ are the Fresnel reflection coefficients which are calculated directly from the transfer-matrix elements in (8) and the parameters $\eta$ of the incidence and transmission media.

$$
r=\frac{\left(m_{11}+m_{12} \eta_{N}\right) \eta_{0}-\left(m_{21}+m_{22} \eta_{N}\right)}{\left(m_{11}+m_{12} \eta_{N}\right) \eta_{0}+\left(m_{21}+m_{22} \eta_{N}\right)} .
$$

The normalized transmitted power through the sensing region of the fiber tip is given by [30]

$$
P_{\text {trans }}=\frac{\int_{0}^{L} d z \int_{\alpha_{1}(z)}^{\alpha_{2}}\left[R_{\mathrm{p}}\right]^{N_{\text {ref }}} I(\theta) d \theta}{\int_{0}^{L} d z \int_{\alpha_{1}(z)}^{\alpha_{2}} I(\theta) d \theta}
$$

where

$$
I(\theta)=\frac{n_{0}^{2} \sin \theta \cos \theta}{\left(1-n_{0}^{2} \cos ^{2} \theta\right)}
$$

is the intensity distribution between the continua of guided modes (assuming meridional excitation). As shown in (11), $R_{p}$ is the reflectivity of the multilayer structure, and $N_{\text {ref }}$ is the number of reflections that the optical ray with the propagation angle $\theta$ undergoes in the sensing region.

In order to evaluate the number of reflections, we consider a length $d L$ of the taper region around the coordinate $z$ where the radius of the taper is $\rho(z)$, as illustrate in Fig. 2.

With regard to this figure, $\tan (\beta)=b / h$ and $\tan [\alpha(z)-\beta]=\left(h_{0}-h\right) / b$, therefore

$$
h=\frac{h_{0}}{1+\tan (\beta) \tan [\alpha(z)-\beta]} .
$$

also

$$
h_{0}=2 \rho(\mathrm{z}) \tan [\alpha(z)-\beta] .
$$

With these relations, the equation for the longitudinal length advanced when the ray propagates from one side of the taper to the other is given by 


$$
h=\frac{2 \rho(z) \tan [\alpha(z)-\beta]}{1+\tan \beta \tan [\alpha(z)-\beta]} .
$$

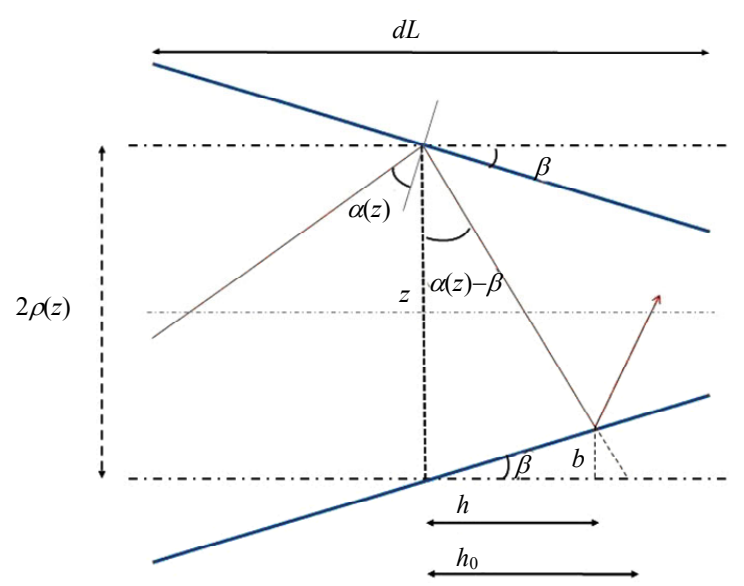

Fig. 2 Schematic of a length $d L$ of the taper region around the coordinate $z$ where the radius of the taper is $\rho(z)$.

The ray is reflected in the lower taper surface and propagates upwards towards the upper taper surface. For simplification, we can consider the same length $h$ is advanced by the ray associated with this upwards propagation (actually, the real value is smaller than $h$ ). Therefore, in the distance $2 h$, we have a reflection, which means in the distance $d L$ the number of reflections is

$$
\delta N=\frac{1+\tan (\beta) \tan [\alpha(z)-\beta]}{4 \rho(z) \tan [\alpha(z)-\beta]} .
$$

Considering the full taper length, the number of reflections can be calculated as

$$
N_{\text {one-path }}=\int_{0}^{L} \frac{1+\tan (\beta) \tan [\alpha(z)-\beta]}{4 \rho(z) \tan [\alpha(z)-\beta]} d z .
$$

Since the configuration works in reflection, the total number of reflections is given by

$$
N_{\text {ref }}=2 N_{\text {one-path }}=\int_{0}^{L} \frac{1+\tan (\beta) \tan [\alpha(z)-\beta]}{2 \rho(z) \tan [\alpha(z)-\beta]} d z
$$

with $\alpha(z)$ given by (2). In order to access the performance of phase interrogation of SPR sensors supported by fiber structures, it is convenient to express the amplitude reflection coefficients $r_{s}$ and $r_{p}$ in the polar form as

$$
r_{s}=\left|r_{s}\right| e^{\mathrm{i} \phi_{s}}, r_{p}=\left|r_{p}\right| e^{\mathrm{i} \phi_{p}} .
$$

If $\left(\delta \phi_{p, s}\right)_{j}=\left[\left(\phi_{\mathrm{p}}-\phi_{\mathrm{s}}\right)_{j}\right]$ is the phase shift of the light between the $p$ and $s$ polarizations for reflection $j$, the total phase shift accumulated by the light that propagates in the optrode is given by

$$
\left(\phi_{p}-\phi_{s}\right)_{\text {total }}=2 \sum_{j=1}^{N_{\text {onc-path }}}\left(\phi_{p}-\phi_{s}\right)_{j}
$$

The situation is somehow simplified if an average phase shift per reflection is determined. At the input of the taper, the angle of incidence can be considered to be $\alpha_{0}$, which corresponds to a phase shift of $\left(\delta \phi_{p, s}\right)_{\alpha 0}=\left(\phi_{p}-\phi_{s}\right)_{\alpha 0}$; at the end of the taper, the phase shift is $\left(\delta \phi_{p, s}\right)_{\alpha(L)}=\left(\phi_{p}-\phi_{s}\right)_{\alpha(L)}$, with $\alpha(L)$ obtained from (2) when $\rho(z)=\rho_{L}$. Therefore, an approximation for this average phase shift is given by

$$
\left(\delta \phi_{p, s}\right)_{\text {average }}=\frac{\left(\delta \phi_{p, s}\right)_{\alpha_{0}}+\left(\delta \phi_{p, s}\right)_{\alpha(L)}}{2},
$$

and the total phase shift accounting for all reflections inside the sensing region is

$$
\Delta \phi_{p, s} \equiv\left(\phi_{p}-\phi_{s}\right)_{\text {total }}=N_{\text {ref }}\left(\delta \phi_{p, s}\right)_{\text {average }} .
$$

At this stage, it is useful to define the sensitivity of the sensing structure to the refractive index $n_{s}$ of the surrounding medium, which can be regarded as the basic parameter quantifying the performance of the sensor. A change in the refractive index $n_{s}=\left(\varepsilon_{s}\right)^{1 / 2}$ of the surrounding dielectric produces a variation in the propagation constant of the surface plasmon, resulting in the modification of the SPR coupling condition. This can be observed as a change in one or more properties of the light transmiting through the sensing structure. The sensitivity can be defined as

$$
S_{n, \xi}=\frac{\delta \xi}{\delta n_{s}}
$$

where $\delta \xi$ is the change in a given property of light (e.g., intensity, resonance wavelength or phase) due to a variation of $\delta n_{\mathrm{s}}$ in the refractive index of the surrounding medium. Here, we particularly concern with the case of phase interrogation, where the property of light of interest is the phase difference $\Delta \phi_{p, s}$ between the $p$ and $s$ polarization components. In this case, the phase sensitivity to refractive index variations is then defined as $S_{n, \phi}=\delta\left(\Delta \phi_{p, s}\right) / \delta n_{s}$. 


\section{Results and discussion}

In this analysis, we consider a multimode silica fiber with the $100-\mu \mathrm{m}$ core diameter with the numerical aperture of 0.24 , tip end diameter of $60 \mu \mathrm{m}$, and sensing region of $L=1 \mathrm{~cm}$. Accordingly, the number of reflections estimated using (20) is $N_{\text {ref }}$ $=60$. The refractive indices for the metal and oxide layers are obtained from experimental data available in literatures [32-35]. Concerning the metallic layer, we have found in a previous study that silver is the most adequate one [36]. Figure 3 shows the spectrum of the light returning from the sensing head for different thicknesses of the silver layer, and when combined with the spectral curves obtained when the oxide layer is included, it turns out the best thickness for the silver layer is $80 \mathrm{~nm}$, so this value is set for the analysis that provides the results delivered in the following.

For the outer oxide layer, three different oxide materials are compared, titanium dioxide $\left(\mathrm{TiO}_{2}\right)$, silicon dioxide $\left(\mathrm{SiO}_{2}\right)$, and aluminum oxide $\left(\mathrm{Al}_{2} \mathrm{O}_{3}\right)$.
It is known that the introduction into this layer modifies substantially the characteristics of the SPR resonance, most notably the spectral region where it appears [37]. Figure 4 shows the location and shape evolution of the resonance for different thicknesses of the oxide layer. The SPR sensing head is assumed to be surrounded by a non-dispersive external medium with the refractive index of $n_{s}=1.33$.

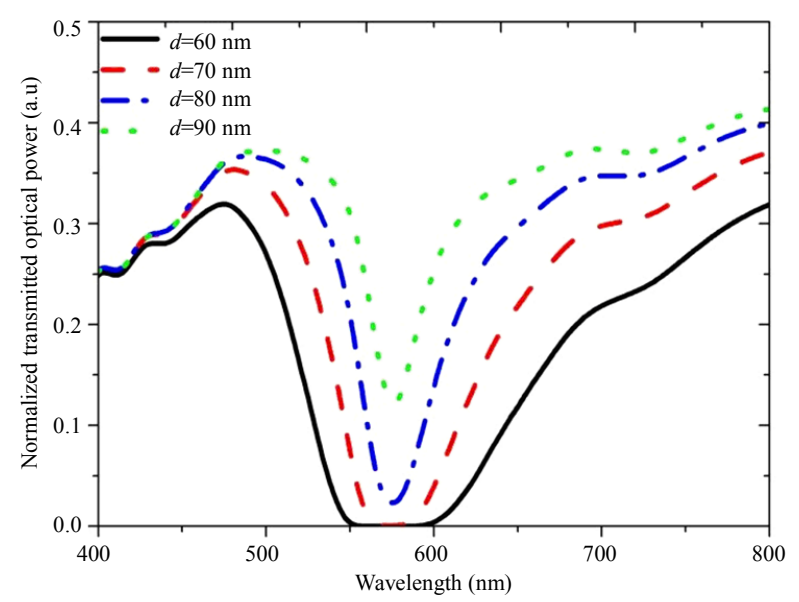

Fig. 3 Spectra of the normalized returned optical power for a fiber optic SPR structure with a single silver layer for several thicknesses (refractive index of the external medium: 1.33).

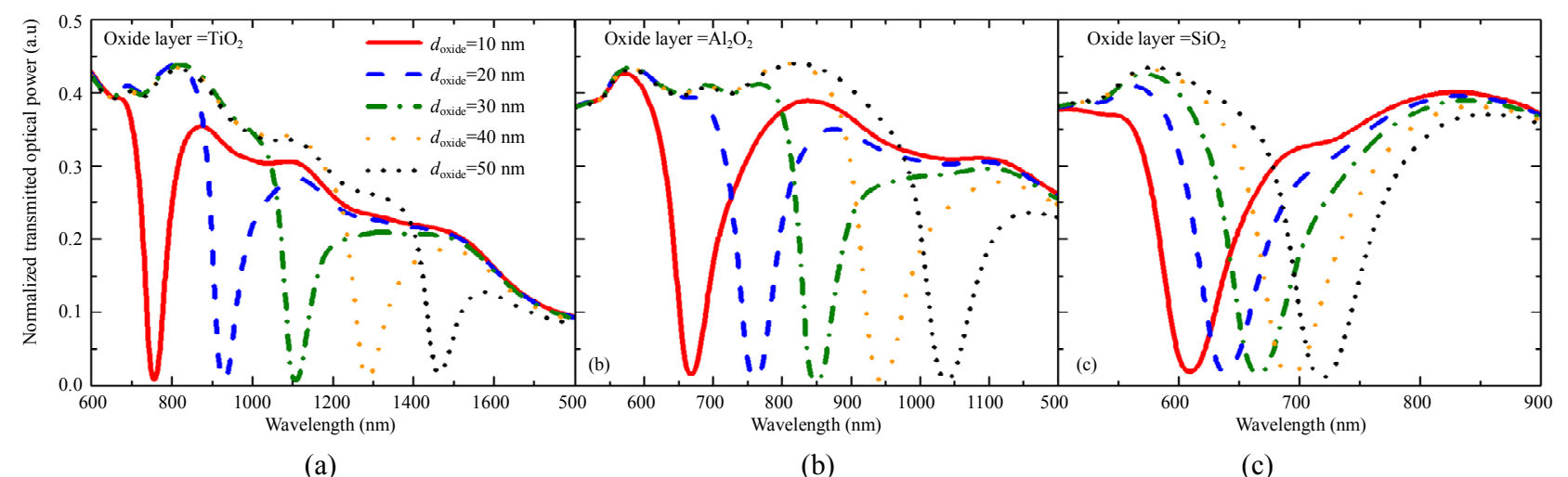

Fig. 4 Spectral behavior of the double-layer fiber optic SPR structure for different thicknesses of the oxide layer $\left(\mathrm{TiO}_{2}, \mathrm{SiO}_{2}\right.$, and $\mathrm{Al}_{2} \mathrm{O}_{3}$ oxide materials are associated with (a), (b), and (c), respectively; refractive index of the external medium: 1.33).

Regarding Fig.4, we observe that inclusion of a $\mathrm{TiO}_{2}$ layer permits the largest displacement of the spectral response of the SPR resonance, making it possible to locate it within the $830 \mathrm{~nm}, 1300 \mathrm{~nm}$, and $1550 \mathrm{~nm}$ telecommunication bands by adjusting the layer thickness within an interval which is technically feasible. Also, there are consequences in the width of the resonance, which consistently becomes larger when it moves to longer wavelengths, as shown in Fig. 5 which displays the spectral width of the resonance versus the thickness of the oxide layer.

To perform the study of phase interrogation of fiber optic based SPR sensing structures, a single oscillation angle of the light in the fiber, associated with a specific propagation mode, shall be 


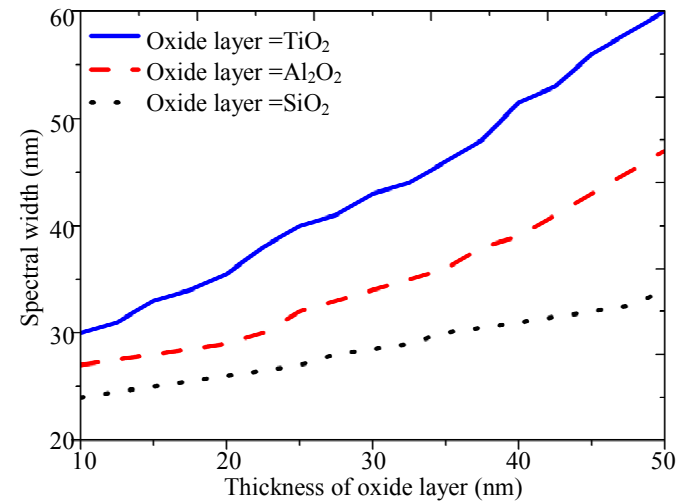

Fig. 5 Evolution of the spectral width of the SPR resonance (full width at the power level $6 \mathrm{~dB}$ above the minimum) as a function of the thickness of the oxide layer (the refractive index of the external medium is considered to be 1.33).

considered (oscillation angle defined by the fiber axis and the light ray). The higher the numerical aperture of the fiber is, the larger this angle can be, meaning a smaller angle of incidence in the interface core-cladding of the fiber. In the fiber length where SPR occurs, this condition favors the introduction into a larger phase difference $\Delta \phi_{p, s}$ between the TM and TE polarizations of the light in the reflection process, increasing the sensitivity to variations of the refractive index of the surrounding medium, $n_{s}$. As mentioned in the prior section, the phase shift introduced in each reflection is assumed constant, the average of phase shift at the beginning of the taper $\left(\delta \phi_{p, s}\right)_{\alpha 0}=\left(\phi_{p}-\phi_{s}\right)_{\alpha 0}$, with $\alpha_{0}=80^{\circ}$, and at the end of the taper $\left(\delta \phi_{p, s}\right)_{\alpha,(L)}=\left(\phi_{p}-\phi_{s}\right)_{\alpha(L)}$, with $\alpha_{L}=73^{\circ}$.

One of the topics investigated in this work is the tuning of the characteristics of the thin film layers to obtain sensor operation in the desired wavelength window. Figure 6 shows the normalized transmittance and phase difference $\Delta \phi_{p, s}$ versus $n_{s}$ for layouts with $\mathrm{TiO}_{2}$ and $\mathrm{Al}_{2} \mathrm{O}_{3}$ layers with the thicknesses of $15 \mathrm{~nm}$ and $30 \mathrm{~nm}$, respectively, which enables to operate the sensor in the first telecommunications window (the data are shown for $\lambda=830 \mathrm{~nm}$; from the data given in Fig. 4(c), this wavelength is not accessible by considering a $\mathrm{SiO}_{2}$ layer). The analysis of the results shows the maximum of the refractive index phase sensitivity, $S_{n, \phi}=\delta\left(\Delta \phi_{p, s}\right) / \delta n_{s}$, occurs close to $n_{s}=1.330$, with a value of $5.72 \times 10^{4}$ degrees/RIU for the case of the titanium dioxide layer, while for the situation of aluminum oxide layer a slightly smaller value is obtained $\left(5.57 \times 10^{4}\right.$ degrees/RIU for $\left.n_{s}=1.327\right)$. In both cases, the maximum phase sensitivity is close to the SPR resonance, where the transmitted optical power is rather low. This means almost no light to be detected, therefore decreasing substantially the signal-to-noise ratio. As such, a compromise is needed so that the phase sensitivity is degraded to a certain extent while the signal-noise-ratio in the detection process increases, therefore reaching a situation of the maximum resolution in the measurement of $n_{s}$.

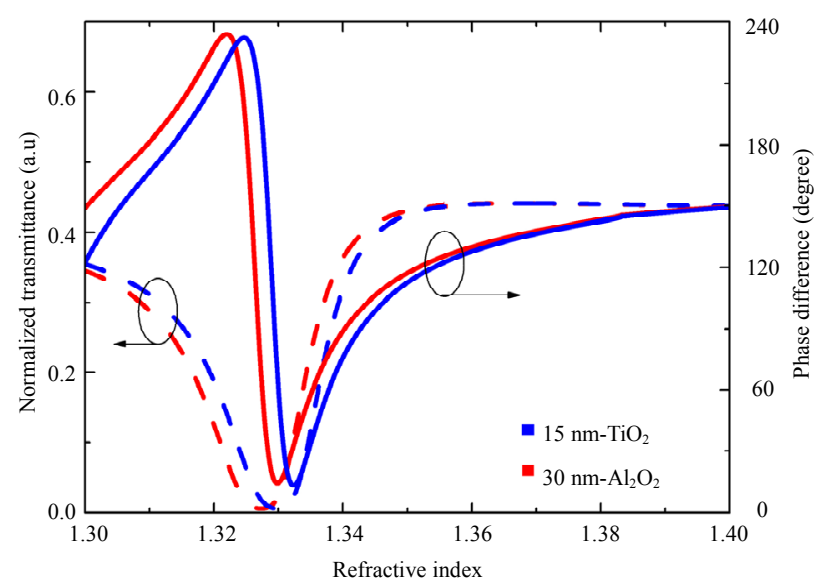

Fig. 6 Normalized transmittance and phase difference (between $p$ and $s$ polarizations) as a function of the refractive index of the surrounding medium for an inner silver layer of $80 \mathrm{~nm}$ combined with a $\mathrm{TiO}_{2}$ layer of $15 \mathrm{~nm}$ (solid curves) and a $\mathrm{Al}_{2} \mathrm{O}_{3}$ layer of $30 \mathrm{~nm}$ (dashed curves) (these thicknesses are considered to allow the operation wavelength around $830 \mathrm{~nm}$ ).

For the sensing head to operate in the second $(1300 \mathrm{~nm})$ and third $(1550 \mathrm{~nm})$ telecommunications spectral windows, the observation of Fig. 4 indicates the need to consider an external layer of $\mathrm{TiO}_{2}$ with thicknesses of $40 \mathrm{~nm}$ and $55 \mathrm{~nm}$, respectively. The normalized transmittance and $\Delta \phi_{p, s}$ versus $n_{s}$ are shown in Fig. 7. Regarding these results, it turns out the maximum phase sensitivity has the value of $4.11 \times 10^{4}$ degrees/RIU (at $n_{s}=1.335$ ) and $3.1 \times 10^{4}$ degrees/RIU (at $n_{s}=1.332$ ) when the interrogation wavelengths are $1300 \mathrm{~nm}$ and $1550 \mathrm{~nm}$, respectively. Due to a wide availability of the high performance optical fiber technology at a relatively 
low cost, considering SPR sensors supported by optical fiber platforms is advantageous to operate within these telecommunications windows, particularly the one in the 1550 -nm region, allowing the design and implementation of sensing structures with rather favorable characteristics.

As mentioned in the above section, since the maximum phase sensitivity occurs in a region close to the dip of the SPR resonance, the available optical power level is rather low, which will degrade the signal-noise ratio to a point that such high sensitivity does not have impact on the final phase reading resolution. Therefore, it shall be considered to operate in a wavelength where there is enough transmitted optical power, which in principle means it can be located in either side of the SPR transmittance curve. To investigate this aspect, the achievable phase sensitivity for different levels of transmittance in both sides of the SPR resonance is analyzed, for the case being selected, which is associated with Fig. 7, i.e., relative to an operating wavelength of $1300 \mathrm{~nm}$. The results obtained are shown in Fig. 8.

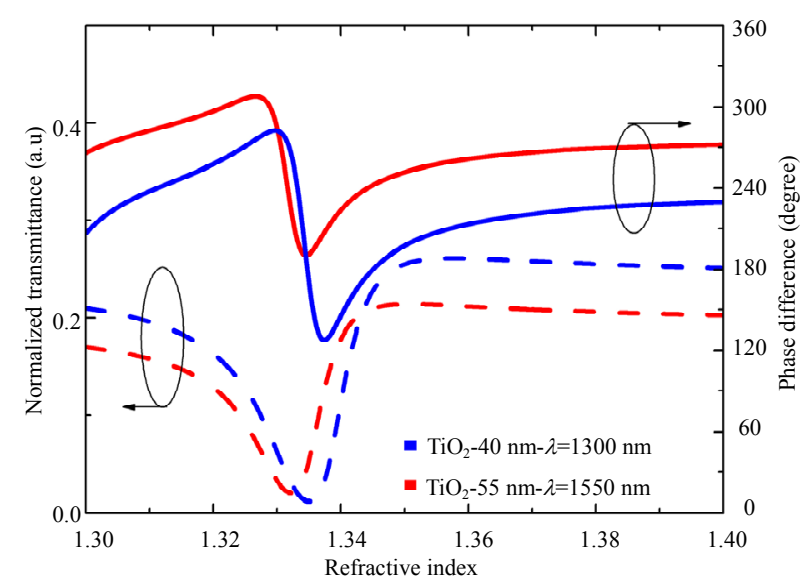

Fig. 7 Normalized transmittance and phase difference (between $p$ and $s$ polarizations) as a function of the refractive index of the surrounding medium for an inner silver layer of $80 \mathrm{~nm}$ combined with a $\mathrm{TiO}_{2}$ layer of $40 \mathrm{~nm}$ (solid curves) and a $\mathrm{TiO}_{2}$ layer of $55 \mathrm{~nm}$ (dashed curves), associated with interrogation wavelengths of $1300 \mathrm{~nm}$ and $1550 \mathrm{~nm}$, respectively.

With the wavelength fixed, different transmittance levels mean different refractive indices of the surrounding medium. As expected from the observation of Fig. 4(a), due to the asymmetry of the SPR resonance, its left side provides limited freedom to the choice of the operating pair (transmittance and sensitivity), indicating the preference to work out in the right side of the resonance.

Optical sensors based on the SPR phenomenon are inherently highly sensitive to variations in the refractive index of the external medium, permitting to detect very small changes in this measurand, for example variations induced by biochemical processes. Therefore, it is in the context of biochemical sensing that these devices are rather appellative, pointing out the relevance of optimizing their performance in the situation where the external medium is water. Consequently, for each type of the oxide layer, the appropriate thickness is determined in order to attain the maximum sensitivity at the refractive index of water evaluated at the sensor operating wavelength.

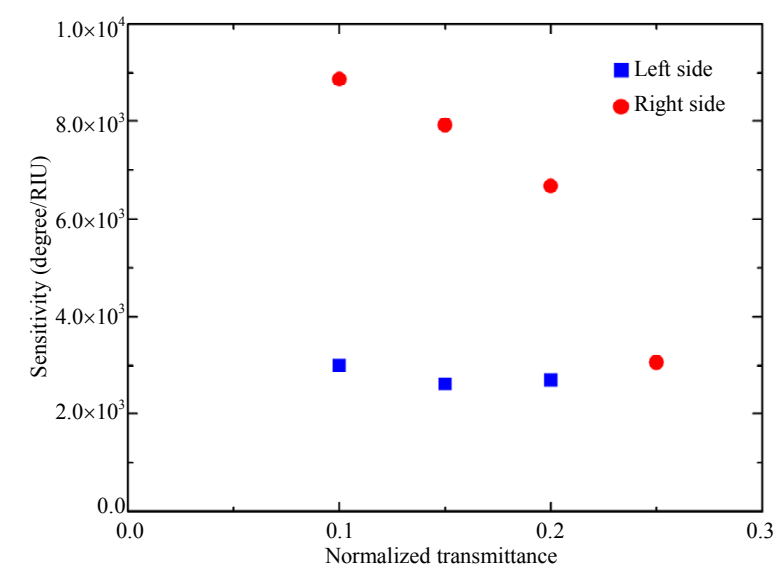

Fig. 8 Phase sensitivity versus normalized transmittance for a fiber optic SPR structure with an inner silver layer of $80 \mathrm{~nm}$ combined with a $\mathrm{TiO}_{2}$ layer of $40 \mathrm{~nm}$ (interrogation wavelength of $1300 \mathrm{~nm})$.

Figure 9 shows the maximum phase sensitivity obtained when the operation wavelengths are $630 \mathrm{~nm}$ [Fig.9(a)] and $830 \mathrm{~nm}$ [Fig.9(b)]. The most immediate outcome of the analysis of this data is that for the case of operation at $630 \mathrm{~nm}$ it is possible to find the required thickness for the oxide materials considered, $\mathrm{TiO}_{2}, \mathrm{SiO}_{2}$, and $\mathrm{Al}_{2} \mathrm{O}_{3}$. However, when moving to $830 \mathrm{~nm}$, the layer of $\mathrm{SiO}_{2}$ leaves to be an 
option, as the results shown in Fig. 4(c) already indicated. The same happens when operating at the wavelengths of $1300 \mathrm{~nm}$ [Fig. 10(a)] and $1550 \mathrm{~nm}$ [Fig. 10(b)], as observed in Fig. 10. This figure also shows the titanium dioxide option for the external layer permits to address operation at both wavelengths, eventually being feasible to consider a layer of aluminum oxide for 1300-nm interrogation, associated with a width of about $86 \mathrm{~nm}$, which is still technically feasible.

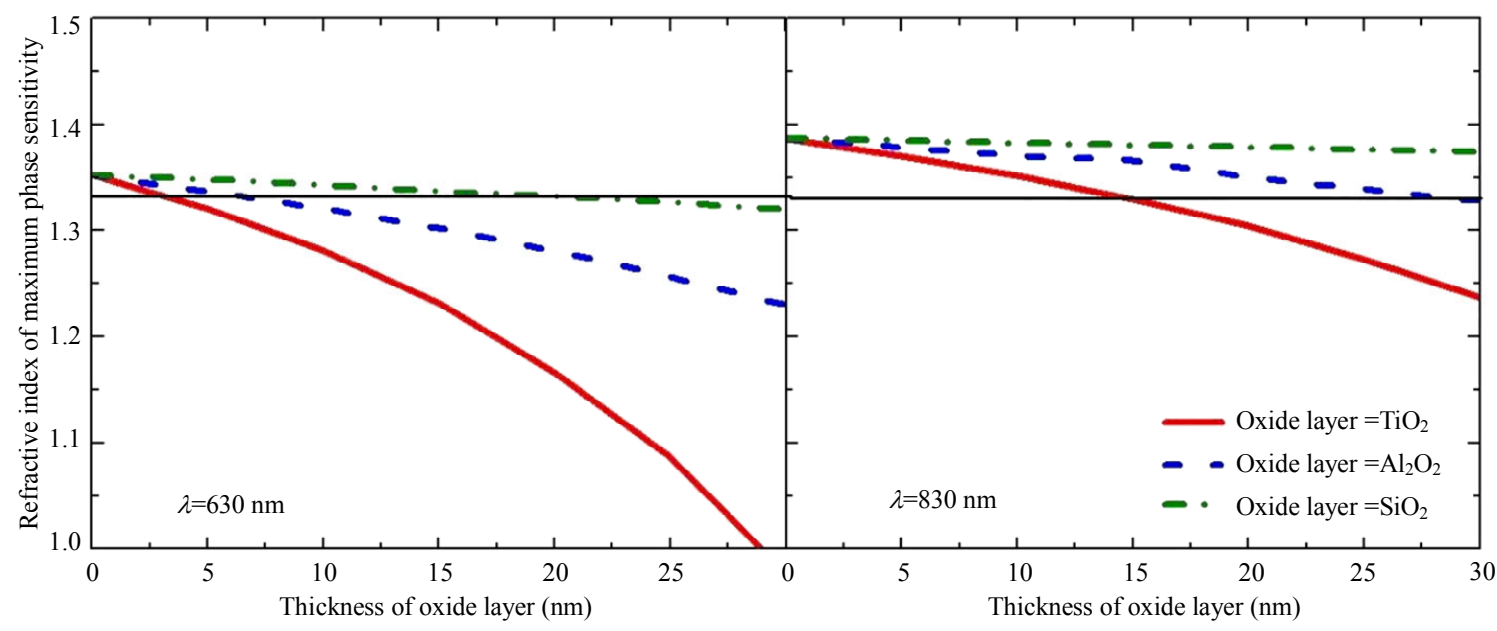

(a)

(b)

Fig. 9 Thickness of the oxide layer required to operate with the maximum sensitivity to changes in the refractive index of water, determined by the intersection of the horizontal line (that defines in the $y$-axis the water refractive index at the wavelength of operation) with the curves associated with different materials for the oxide layer [the interrogation wavelengths are $630 \mathrm{~nm}$ (a) and $830 \mathrm{~nm}$ (b)].

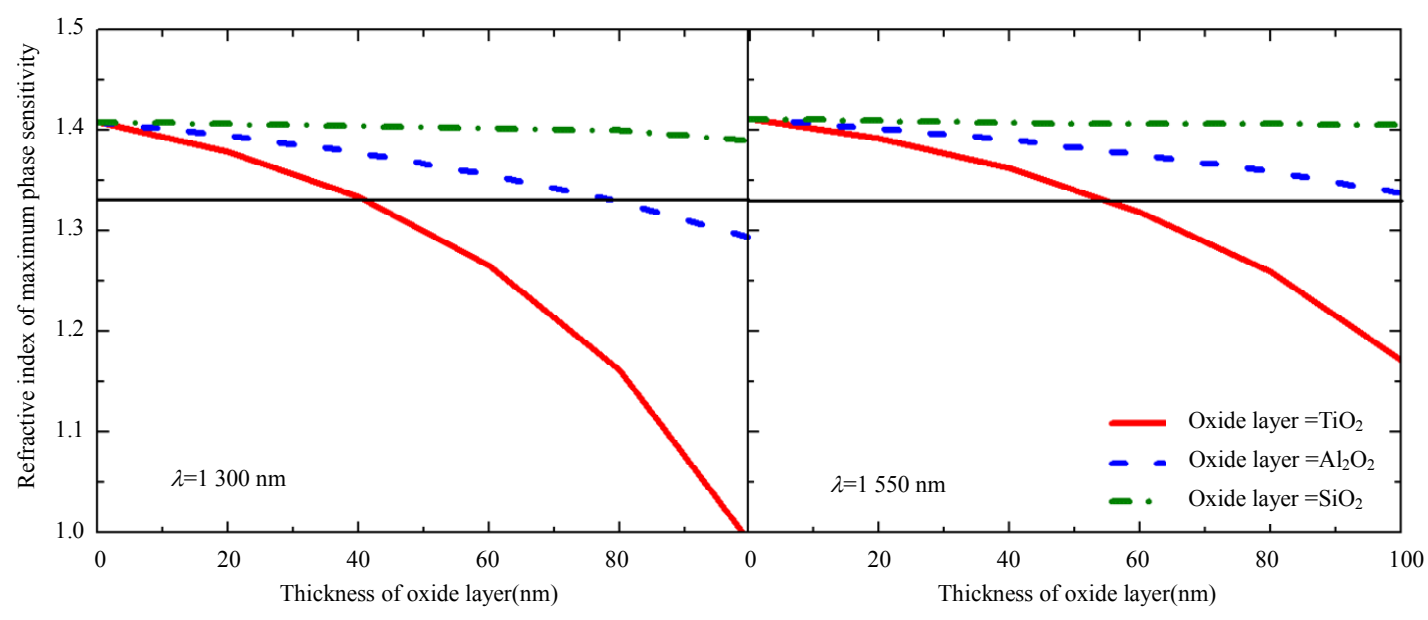

(a)

(b)

Fig. 10 Same as Fig. 9 but now with interrogation wavelengths of $1300 \mathrm{~nm}$ (a) and $1550 \mathrm{~nm}$ (b).

\section{Final remarks}

In this work, an optrode sensing structure based on a linearly tapered fiber tip, covered by a double layer thin film which induces plasmonic behavior at a specific wavelength range, is researched. The characteristics of the plasmonic resonance depend on the refractive index of the surrounding medium, therefore information is obtained on this parameter performing phase interrogation at a specific wavelength. The inner component of the double layer is metallic (silver), while the external one is an oxide dielectric layer. Two main characteristics of this sensing layout are on target: one is the possibility to tune the sensor sensitivity to variations of the refractive index of the surrounding medium, 
and the other is the amenability to tune the measurement operation to a specific wavelength region, most notably the one around $1550 \mathrm{~nm}$ to benefit from the large portfolio of high performance fiber optic components available at these wavelengths. It is found the inclusion of an internal silver layer with the 80 -nm-thickness permits the better performance for any of the three different oxide materials considered for the external layer. Also, the results obtained shows the better flexibility is provided by the inclusion of a layer of titanium dioxide, and the approach is compatible with tuning the sensor operation to a large wavelength window that includes operationally important wavelengths $(630 \mathrm{~nm}, 830 \mathrm{~nm}, 1300 \mathrm{~nm}$, and $1550 \mathrm{~nm})$.

The experimental validation of this study can be done by the application of techniques developed along with the years for the interrogation of polarimetric fiber sensing interferometers illuminated by monochromatic light [38]. Following these approaches, laser light is injected into the multimode fiber at an angle such that the selected core mode is excited. At the input of the sensing head, a fiber polarizer element shall be included in order to have light in the TE and TM polarizations, recombined at the output of the measurement region by means of a second polarizer. This will generate an interferometric signal with a phase proportional to the optical path difference accumulated by the propagation of the TE and TM polarizations, therefore including the phase variations of the light in the TM polarization induced by changes in the surrounding refractive index.

To recover the phase of this signal, a robust and sensitive technique involves the addition to the interferometric phase of a sinewave component, which can be generated by sinewave modulation of the wavelength of the laser light. After photodetection and incorporation of signal processing, which includes a feedback loop to the laser source to tune the direct current (DC) value of the light wavelength, the information on the quasi-static phase of the polarimetric sensing interferometer, therefore on the refractive index of the external medium, can be obtained from the feedback signal [39]. This approach is compatible with a DC phase resolution of the order of $1 \mathrm{mrad}$, which means from the results presented here refractive index measurement resolutions of the order $10^{-7}$ shall be possible.

The analysis presented in this work permits to guide the design of fiber optrodes highly sensitive to the surrounding medium refractive index by plasmonic interaction at a certain wavelength window and considering phase reading. These optrodes incorporate an internal metallic layer (Ag) covered with an oxide layer. Different oxide materials $\left(\mathrm{TiO}_{2}, \mathrm{SiO}_{2}\right.$, and $\left.\mathrm{Al}_{2} \mathrm{O}_{3}\right)$ are addressed, and the main characteristics of these structures for refractive index sensing are determined. Paths for the experimental investigation of these sensing devices are also briefly introduced.

\section{Acknowledgment}

This research was performed in the framework of Project PTDC/FIS/119027/2010 (Plasmonics Based Fiber Optic Sensing with Enhanced Performance) funded by National Funds through FCT - Fundação para a Ciência e a Tecnologia. L. Coelho acknowledges the support from FCT grant SFRH/BD/78149/2011.

Open Access This article is distributed under the terms of the Creative Commons Attribution 4.0 International License (http://creativecommons.org/ licenses/by/4.0/), which permits unrestricted use, distribution, and reproduction in any medium, provided you give appropriate credit to the original author(s) and the source, provide a link to the Creative Commons license, and indicate if changes were made.

\section{References}

[1] J. L. Santos and F. Farahi, Handbook of optical sensors. BocaRaton: CRC Press, 2014.

[2] J. Janata, Principles of chemical sensing. Berlin: Springer-Verlag, 2009. 
[3] F. S. Ligler and C. R. Taitt (Editors), Optical biosensors: today and tomorrow. Hungary: Elsevier, 2011.

[4] J. G. Webster and H. Eren (Editors), Measurement, instrumentation and sensors handbook. BocaRaton: CRC Press, 2014.

[5] R. W. Wood and P. Mag, "On a remarkable case of uneven distribution of light in a diffraction grating spectrum," Proceedings of the Physical Society of London, 1902, 4: 396-402.

[6] L. Rayleigh, "On the dynamical theory of gratings," Proceedings of the Royal Society of London A Mathematical Physical \& Engineering Sciences, 2010, 55(532): 399-416.

[7] U. Fano, "The theory of anomalous diffraction gratings and of quasi-stationary waves on metallic surfaces (sommerfeld's waves)," Journal of the Optical Society of America, 1941, 31(3): 213-222, 1941.

[8] A. Otto, "Excitation of nonradiative surface plasma waves in silver by method of frustrated total reflection," Zeitschrift fur Physik A Hadrons \& Nuclei, 1968, 216(4): 398-410.

[9] E. Kretschmann and H. Reather, "Notizen: radiative decay of non radiative surface plasmons excited by light," Zeitschrift fur Naturforschung A, 2014, 23(12), 2135-2136.

[10] H. A. Atwater, "The promise of plasmonics," Scientific American, 2007, 296(4): 56-62.

[11] R. C. Jorgenson and S. S. Yee, "A fiber-optic chemical sensor based on surface plasmon resonance," Sensors and Actuators B: Chemical, 1993, 12(3): 213-220.

[12] S. P. Resurrection, "Surface plasmon resurrection," Nature Photonics, 2012, 6(11): 707-794.

[13] H. Raether, Surface plasmons on smooth and rough surfaces and on gratings. Berlin: Springer-Verlag, 1988.

[14] J. Homola, S. S. Yee, and G. Gauglitz, "Surface plasmon resonance sensors: review," Sensors and Actuators B: Chemical, 1999, 54(1-2): 3-15.

[15] J. Homola, "Present and future of surface plasmon resonance biosensors," Biosystems, 2003, 377(3): 528-539.

[16] H. Suzuki, M. Sugimoto, Y. Matsui, and J. Kondoh, "Effects of gold film thickness on spectrum profile and sensitivity of a multimode-optical-fiber SPR sensor," Sensors and Actuators B: Chemical, 2008, 132(1): 26-33.

[17] S. K. Ozdemir and G. Turhan-Sayan, "Temperature effects on surface plasmon resonance: design considerations for an optical temperature sensor," Journal of Lightwave Technology, 2003, 21(3): 805-814.

[18] H. R. Gwon and S. H. Lee, "Spectral and angular responses of surface plasmon resonance based on the
Kretschmann prism configuration," Materials Transaction, 2010, 51(5): 1150-1155.

[19] Y. C. Li, Y. F. Chang, L. C. Su, and C. Chou, "Differential-phase surface plasmon resonance biosensor," Analytical Chemistry, 2008, 80(14): 5590-5595.

[20] H. P. Chiang, J. L. Lin, and Z. W. Chen, "High sensitivity surface plasmon resonance sensor based on phase interrogation at optimal wavelengths," Applied Physics Letters, 2006, 88(14): 141105-1-141105-3.

[21] S. P. Ng, C. M. L. Wu, S. Y. Wu, and H. P. Ho, "White-light spectral interferometry for surface plasmon resonance sensing applications," Optics Express, 2011, 19(5): 4521-4527.

[22] A. K. Sharma, R. Jha, and B. D. Gupta, "Fiber-optic sensors based on surface plasmon resonance: a comprehensive review," IEEE Sensors Journal, 2007, 7(8): 1118-1129.

[23] A. K. Sharma and B. D. Gupta, "On the performance of different bimetallic combinations in surface plasmon resonance based fiber optic sensors," Journal Applied Physics, 2007, 101(9): 093111-1-093111-6.

[24] R. Slavik, J. Homola, and J. Ctyroky, "Miniaturization of fiber optic surface plasmon resonance sensor," Sensors and Actuators B: Chemical, 2008, 51(1-3): 311-315.

[25] B. D. Gupta and C. D. Singh, "Evanescentabsorption coefficient for diffuse source illumination: uniform- and tapered-fiber sensors," Applied Optics, 1994, 33(13): 2737-2742.

[26] B. Grunewald and G. Holst, "Fiber optic refractive index microsensor based on white-light SPR excitation," Sensors and Actuators A: Physical, 2004, 113(2): 174-180.

[27] Y. C. Kim, W. Peng, S. Banerji, and K. S. Booksh, "Tapered fiber optic surface plasmon resonance sensor for analyses of vapor and liquid phases," Optics Letter, 2005, 30(17): 2218-2220.

[28] M. Niggemann, A. Katerkamp, M. Pellmann, P. Bolsmann, J. Reinbold, and K. Cam-mann, "Remote sensing of tetrachloroethene with a micro-fibre optical gassensor based on surface plasmon resonance spectroscopy," Sensors and Actuators B: Chemical, 1996, 34(1-3): 328-333.

[29] L. A. Obando and K. S. Booksh, "Tuning dynamic range and sensitivity of white-light, multimode, fiber-optic surface plasmon resonance sensors," Analytical Chemistry, 1999, 71(22): 5116-5122.

[30] Y. Yinquan, H. Die, H. Li, and L. Min, "Theoretical investigations for surface plasmon resonance based optical fiber tip sensor," Sensors and Actuators B: Chemical, 2013, 188(11): 757-760.

[31] M. Born and E. Wolf, Principles of optics, seventh (expanded) edition. Cambridge: Cambridge 
University Press, 1999.

[32] P. Winsemius, F. F. Van Kampen, H. P. Lengkeek, and C. G. Van Went, "Temperature dependence of the optical properties of $\mathrm{Au}, \mathrm{Ag}$ and $\mathrm{Cu}$," Surface and Coatings Technology, 2001, 6(8): 1220-1224.

[33] J. R. DeVore, "Refractive index of rutile and sphalerite," Journal of the Optical Society of America, 1951, 41(6): 416-419.

[34] E. D. Palik, Handbook of optical constants of solids. Boston: Academic Press, 1995.

[35] I. H. Malitson, "Interspecimen comparison of the refractive index of fused silica," Journal of the Optical Society of America, 1965, 55(10), 1205-1208.

[36] H. Moayyed, I. Leite Teixeira, L. Coelho, J. L.
Santos, and D. Viegas, "Analysis of phase interrogated SPR fiber optic sensors with bimetallic layers," IEEE Sensors Journal, 2014, 14(10): 3662-3668.

[37] B. Priya and B. D. Gupta, "Surface-plasmonresonance-based fiber-optic refractive index sensor: sensitivity enhancement," Applied Optics, 2011, 50(14): 2032-2036.

[38] B. Culshaw, "Interferometric optical fibre sensors," IETE Journal of Research, 1986, 32(4): 311-318.

[39] C. J. Misas, F. M. Moita. Araujo, L. A. Ferreira, J. L. Santos, and J. M. López-Higuera, "Interrogation of low-finesse Fabry-Pérot cavities based on modulation of the transfer function of a wavelength division multiplexer," Journal Lightwave Technology, 2001, 19(5): 673-681. 